\title{
Inter-trial response strategy in operative time estimation
}

\author{
M. J. ELLIS AND M. G. WADE, CHILDREN'S RESEARCH \\ CENTER, UNIVERSITY OF ILLINOIS, Champaign, Ill. 61820
}

Two strategies "creeping" and "bracketing" were postulated as alternative methods of approximating a target score, and it is suggested that the use of these strategies is influenced by the conditions under which performance took place. This was tested using large and small movements of a cursor along a trackway against large and small resistances. Each $S$ 's scores were autocorrelated, lagged once and lagged twice while learning and while continuing to respond in the absence of $K R$. Analysis of the variance of the transformed auto-correlations showed them to be significantly more positive for the large movement when lagged once. It was concluded that intertrial strategy was influenced by the learning conditions, and that the Ss used only the preceding trial in formulating their strategy for the next.

The analysis of responses themselves and the strategies adopted by Ss to increase performance are becoming necessary to study the learning process. The nature of the response itself has been extensively studied in the research on tracking behavior. The generation of graphic or electrical analogs of the response have led to the development of methods whereby one time-varying trace is correlated with another (Blackman \& Tukey, 1959; Sollberger, 1965; Fitts, Bennett, \& Bahrick, 1956). In many cases an input analog is cross-correlated with the response analog, and less usually, the response analog is auto-correlated, i.e., with itself. This leads to an auto-correlation of unity, but if the second of the paired response analogs is delayed, then the auto-correlation reflects the relation of one response on that which follows after the lag.

The auto-correlation can also be computed on discrete rather than continuous responses. This is an uncommon procedure but a potentially useful one since the effect of separate trials on others can be determined collectively (Michon, 1962). Thus a series of trials could be considered analogous to a digitized series of samples from a wave form. Serial effects during learning reflect the strategy employed by the $S$ in treating a response in terms of the preceding trial.

Simplifying this situation, there seem to be two strategies available to the S: One strategy was aptly described by Michon (1967, p. 71) as "creeping," and the other is described here as "bracketing." A creeping strategy involves the use of successive reductions in the error so that the target response is approached from one direction. The bracketing strategy involves hunting behavior whereby $S$ utilizes responses that have errors that alternate in sign. The $S$ approaches the target by progressively reducing the magnitude of the errors. In both cases learning results in a distribution of random errors about a mean that is on target.

The auto-correlation computed on a long series of trials differentiates these two strategies. In creeping, each score will tend to be like its predecessor with the exception of an added correction factor and an error component. Thus, the autocorrelation will be positive. During bracketing, successive scores will tend to lie on opposite sides of the mean and the successive scores will be unlike each other. A negative auto-correlation will result.

This study employed data from an experiment already reported by Ellis, Schmidt, \& Wade (in press). They showed that the presence of a large movement in an operative time estimation task enabled the Ss to learn more quickly and to exhibit smaller absolute errors. The purpose of this study was to determine whether the Ss in the large movement condition used a different strategy from those in a minimal movement condition. Autocorrelation lagged once will reveal the collective relation of trials on ensuing trials and auto-correlation lagged twice will show whether serial effects bridged an intervening trial. A further comparison determined whether the removal of knowledge of results (KR) reduced the $S$ to responding stochastically.

Method. The apparatus and methods have been reported in full (Ellis, Schmidt, \&. Wade, in press). The motor response required the $S$ to learn to fill a 2-sec interval with the movement of a cursor along a trackway.

The $S$ was seated facing the trackway which was mounted on the front edge of a table-like structure. The distance moved and the resistance to movement were varied independently. The two levels for movement and resistance, the two dimensions in a 2 by 2 crossed design, were 2.5 and $65.0 \mathrm{~cm}$ and minimal and $19.52 \mathrm{~kg}$, respectively. The Ss were given trials at $15-\mathrm{sec}$ intervals in blocks of five separated by $1 \mathrm{~min}$. For the first 30 trials the Ss were given their lapsed times to $.01 \mathrm{sec}$, and for a further 30 trials no scores were reported.

Eighty male undergraduates from the University of Illinois were randomly assigned to four groups of 20 . Scores for the trials with and without KR were treated separately. The two sets of 30 scores for each $S$ were auto-correlated with a lag of one and two trials. The resulting auto-correlations were standardized using Fisher's $z$ ' transformation. Then each of the categories of correlations, lagged once or twice, with or without $\mathrm{KR}$, was subjected to a movement by resistance ( 2 by 2 ) between Ss analysis of variance.

Results. The only significant effect on the auto-correlation was the presence of $65 \mathrm{~cm}$ movement during the responses with KR when the auto-correlation was lagged once $(F=7.20, \mathrm{df}=1 / 76, \mathrm{p}$ $<.01$ ). The mean auto-correlations were .35 and .17 for responses with a $65.0 \mathrm{~cm}$ and $2.5 \mathrm{~cm}$ response, respectively.

The significantly higher auto-correlation for the conditions involving a large movement indicated that there was a consistent tendency for those Ss to produce responses that were serially more similar. This suggests that the Ss in these conditions tended to use a creeping strategy while learning. The minimal movement conditions demonstrated a significantly larger number of negative auto-correlations (observed frequencies of negative $\mathrm{r}=2: 12$, $\chi^{2}=5.78, p<.05$ ) suggesting a tendency towards a bracketing strategy at least in some Ss. The absence of treatment effects when the auto-correlation was lagged twice suggested that the $S$ utilized the trial immediately preceding as the basis on which to formulate his response strategy for the next trial. The mean auto-correlations when lagged twice dropped to $\cdot 16$ and $\cdot 08$, as would be expected from evidence on the serial correlation (Jones, 1966).

The absence of KR removed the effects of the presence of the $65 \mathrm{~cm}$ movement. This was unexpected since the only strategy available to the $S$ would appear to be the reproduction of the previous response as the best estimate of the previously established habit. Therefore, the absence of KR seemed to reduce all groups to a stochastic response strategy.

Discussion. This study showed that the intertrial strategy could be influenced by the conditions under which the learning took place, and that Ss tended to restrict themselves to use of the preceding response in formulating that strategy.

Our method has potential for studying intertrial response strategies as effected by experimental treatments, and the interference effects of interpolated trials. It would seem that auto-correlations on a control group are necessary to enable the $E$ to remove serial correlation effects from consideration. If strategy is to be studied difficult tasks are indicated, since after a performance asymptote is reached the auto-correlation becomes a measure of the variability of the S's responses, there being no requirement for the $S$ to hunt the target. Finally a long series of trials are necessary to increase the reliability of the auto-correlation.

\section{REFERENCES}

BLACKMAN, R. B., \& TUKEY, J. W. The measurement of power spectra. New York: Dover, 1959.

ELLIS, M. J., SCHMIDT, R. A., \& WADE, M. G. Proprioceptive feedback as a determiner of lapsed time estimation. Ergonomics, in press.

FITTS, P. M., BENNETT, W. P., \& BAHRICK, H. Application of autocorrelation analysis to the study of tracking behavior. In G. Finch \& F. Cameron (Eds.), Symposium on Air Force human engineering, personnel and training research. Washington: National Academy of Sciences, National Research Council, Publication No. 455, 1956. Pp. 125-141.

JONES, M. Individual differences. In E. A. Bilodeau (Ed.), Acquisition of skill. New York: Academic Press, 1966. Pp. 112-144.

MICHON, J. A. Timing in temporal tracking. Soesterberg, The Netherlands: Institute for perception RVO-TNO, National Defense Research Org., 1967. SOLLBERGER, A. Biological rhythm research. Amsterdam: Elsevier Publishing Co., 1965. 